\title{
LINES OF MINIMA WITH NO END IN THURSTON'S BOUNDARY OF TEICHMÜLLER SPACE
}

\author{
YUKI IGUCHI
}

\begin{abstract}
Let $\nu^{+}$and $\nu^{-}$be two measured laminations which fill up a hyperbolic surface. Kerckhoff [Duke Math. J. 65 (1992), 187-213] defines a line of minima as a family of surfaces where convex combinations of the hyperbolic length functions of $\nu^{+}$and $\nu^{-}$are minimum. This is a proper curve in the Teichmüller space. We show that there exists a line of minima which does not converge in the Thurston compactification of the Teichmüller space of a compact Riemann surface. We also show that the limit set of the line of minima is contained in a simplex on the Thurston boundary.
\end{abstract}

\section{INTRODUCTION}

Let $X$ be a Riemann surface of type $(g, n)$. That is, $X$ is obtained from a closed Riemann surface of genus $g$ by removing $n$ points. We assume that the Euler characteristic of $X$ is negative and denote by $T(X)$ the Teichmüller space of $X$. In this paper, we investigate the asymptotic behavior of paths in $T(X)$ which were proposed by Kerckhoff $\left[\mathrm{Ke}\right.$. Let $\nu^{+}, \nu^{-}$be two measured geodesic laminations on $X$ and let $\ell\left(\nu^{+}\right)$and $\ell\left(\nu^{-}\right)$be the hyperbolic length functions of $\nu^{+}$and $\nu^{-}$. Kerckhoff showed that there exists a unique hyperbolic surface at which the function $\ell\left(\nu^{+}\right)+\ell\left(\nu^{-}\right)$is minimum over all $T(X)$ if $\nu^{+}$and $\nu^{-}$fill up $X$. Scaling $\nu^{+}$by $e^{t / 2}$ and $\nu^{-}$by $e^{-t / 2}$ and tracing a unique minimizing point $\mathcal{L}_{t}$ of the sum of the length functions, we can consider a one-parameter family $\mathcal{L}=\left\{\mathcal{L}_{t}\right\}_{t \in \mathbb{R}}$ of hyperbolic surface. This family is called a line of minima. It is known that there exists a line of minima between any two distinct points in $T(X)$ (see $[\mathrm{Ke}$ ). It is also known that every line of minima is a uniformly quasi-geodesic for the Teichmüller distance $d_{T}$ (see [CRS1]); namely, there exist universal constants $c \geq 1$ and $C \geq 0$ such that the inequality

$$
\frac{1}{c}|t-s|-C \leq d_{T}\left(\mathcal{L}_{s}, \mathcal{L}_{t}\right) \leq c|t-s|+C
$$

holds for all $s, t \in \mathbb{R}$. There is another significant class of curves in $T(X)$. What is called Teichmüller geodesics are geodesics for the Teichmüller distance. These also have an analytic description similar to lines of minima: Gardiner-Masur GM] showed that for two transverse measured foliations $F$ and $G$, the function defined to be the product of the extremal length functions of $F$ and $G$ is minimized along

Received by the editors August 2, 2011.

2010 Mathematics Subject Classification. Primary 30F45, 30F60, 32G15, 57M15; Secondary 57M50, 32G15, 30F60, 30F45.

Key words and phrases. Teichmüller space, Thurston's boundary, Teichmüller geodesic, line of minima.

The author is partially supported by "Global COE: Computationism as a Foundation for the Sciences". 
a unique Teichmüller geodesic and that this Teichmüller geodesic is defined by a holomorphic quadratic differential whose horizontal and vertical foliations are in the projective measured classes of $F$ and $G$, respectively.

Teichmüller space can be defined either as a space of equivalence classes of conformal structures on $X$ or as a space of equivalence classes of hyperbolic structures on $X$. Each of the two curves considered above is natural from one of these points of view: Tecihmüller geodesics from the point of view of conformal geometry and lines of minima from the point of view of hyperbolic geometry. There is no obvious way to compare the two curves. It is of interest to formulate the convergence of Teichmüller geodesics or lines of minima. There is a natural compactification of $T(X)$ due to Thurston in view of hyperbolic geometry; the action of the mapping class group extends continuously to the compactification and the boundary, called the Thurston boundary, is equal to the set $\mathcal{P} \mathcal{M L}$ of all projective measured laminations. In [DS], Diaz and Series studied the behavior of lines of minima near the Thurston boundary. They showed the following:

Theorem A. Let $\nu^{+}=\sum_{i=1}^{N} a_{i} \alpha_{i}$ be a rational measured lamination (that is, $\alpha_{i}$ is a collection of disjoint simple closed curves on $X$ and $a_{i}>0$ for all $i$ ) and $\nu^{-}$ any measured lamination so that $\nu^{+}, \nu^{-}$fill up the surface. Then

$$
\lim _{t \rightarrow \infty} \mathcal{L}_{t}=\left[\alpha_{1}+\cdots+\alpha_{N}\right] \in \mathcal{P} \mathcal{M} \mathcal{L} .
$$

If $\nu^{+}$and $\nu^{-}$are two measured laminations which fill up the surface and such that $\nu^{+}$is uniquely ergodic and maximal, then

$$
\lim _{t \rightarrow \infty} \mathcal{L}_{t}=\left[\nu^{+}\right] \in \mathcal{P} \mathcal{M} \mathcal{L}
$$

It is worth pointing out that in the case of $\nu^{+}=\sum_{i=1}^{N} a_{i} \alpha_{i}$, the line of minima converges to $\left[\sum_{i=1}^{N} \alpha_{i}\right]$, rather than to the projective class $\left[\sum_{i=1}^{N} a_{i} \alpha_{i}\right]$ of $\nu^{+}$. Similar results on the behavior of Teichmüller geodesics has been proven by Masur Ma. In this case, a geodesic ray is determined by a base surface $X$ and a quadratic differential $q$ on $X$. Roughly speaking, the end of this ray depends on the horizontal foliation $F$ of $q$. Masur showed the following:

Theorem B. If $q$ is a Jenkins-Strebel differential, that is, if $F$ has only compact leaves, then the associated ray converges in the Thurston boundary to the barycenter of the leaves (the foliation with the same closed leaves all of whose cylinders have unit height), while if $q$ is uniquely ergodic and minimal, it converges to the projective class of $F$.

The question of the description of the behavior of an arbitrary Teichmüller geodesic or line of minima in the Thurston boundary is still open. Recently, Lenzhen [L] showed the following:

Theorem C. There exists a Teichmüller geodesic ray which does not converge in the Thurston compactification.

In the introduction of CRS2, Choi, Rafi and Series expected that the above theorem is true for lines of minima. We give a proof for their expectation:

Theorem 1.1. There exists a line of minima which does not converge in the Thurston compactification. 
In the proof of Theorem C, Lenzhen constructed an explicit quadratic differential and flat surface. To prove Theorem 1.1, we show that the line of minima associated to Lenzhen's quadratic differential does not approach any point of the Thurston boundary. Lenzhen mentioned only the case of genus two, but her construction can be extended for the general case. We shall explain the construction precisely in $\S 6$. Moreover, we give a description of the limit set of the line of minima.

This paper is organized as follows. The aim is to prove Theorem 1.1, In $\S 2$, we recall the definitions and properties of ingredients of Teichmüller theory and Thurston theory. In order to prove the theorem, we need to estimate the Teichmüller distance between Teichmüller geodesics and lines of minima in the thin part of the Teichmüller space. For this purpose, Minsky's product region theorem in Mi] and length estimates for short geodesics in CRS2 play an important role. We shall introduce these precisely throughout $\S 3, \S 4$ and $\S 5$. In $\S 6$, we give a proof of Theorem 1.1. In $\S 7$, we investigate limit sets of lines of minima associated to Lenzhen's quadratic differentials.

\section{Preliminaries}

In this section, we describe basic facts of Teichmüller theory and Thurston theory of projective measured laminations. In what follows, $X$ is a Riemann surface of type $(g, n)$. That is, $X$ is obtained from a closed Riemann surface of genus $g$ by removing $n$ marked points. We say that each of the marked points is a puncture.

2.1. Measured laminations. A collection $\gamma$ of disjoint simple geodesics on $X$ is a geodesic lamination on $X$ if the union $|\gamma|$, called the support, of all geodesics in $\gamma$ is a closed set in $X$. Each of the geodesics in $\gamma$ is called a leaf of $\gamma$. Furthermore, let $\Lambda$ be the set of all compact arcs which are transverse to $|\gamma|$ with endpoints, if any, on $X \backslash|\gamma|$. A function $\nu: \Lambda \rightarrow \mathbb{R}$ is a transverse measure on $\gamma$ if the following three properties hold:

(1) $\nu(\alpha)=\nu(\beta)$ if $\alpha$ is isotopic to $\beta$ through elements of $\Lambda$,

(2) $\nu(\alpha)=\sum_{i \in I} \nu\left(\alpha_{i}\right)$ if $\alpha=\bigcup_{i \in I} \alpha_{i}$ and

(3) $\nu(\alpha)>0$ if and only if $\alpha \cap|\gamma| \neq \emptyset$,

for $\alpha, \beta \in \Lambda$ and for any countable family $\left\{\alpha_{i}\right\}_{i \in I}$ of elements of $\Lambda$ which satisfies that $\alpha_{i} \cap \alpha_{j}=\partial \alpha_{i} \cap \partial \alpha_{j}$ if $i \neq j$. A pair $(\gamma, \nu)$ of a geodesic lamination $\gamma$ and a transverse measure $\nu$ supported on $|\gamma|$ is called a measured lamination on $X$. When $\gamma$ is not noted, we abuse notation and abbreviate $(\gamma, \nu)$ to $\nu$ for simplicity. Two measured laminations $\mu$ and $\nu$ are said to fill up $X$ if $i(\mu, \xi)+i(\xi, \nu)>0$ for any measured laminations $\xi$. The set of all measured laminations is denoted by $\mathcal{M L}=\mathcal{M L}(X)$. The positive numbers $\mathbb{R}_{+}$naturally acts on $\mathcal{M L} \backslash\{0\}$ as multiplicative factors, and we denote the quotient set by $\mathcal{P} \mathcal{M L}=\mathcal{P} \mathcal{M L}(X)$.

The simplest example of measured laminations is the case where the supports consist of finitely many compact geodesics. Namely, $\gamma$ is a collection $\left\{C_{j}\right\}_{j \in J}$ of finitely many disjoint simple closed geodesics and $\nu$ is defined to be

$$
\nu(\alpha)=\sum_{j \in J} a_{j} \operatorname{card}\left(\alpha \cap \mathrm{C}_{\mathrm{j}}\right)
$$

for some weight $a_{j}>0$. Writing $\mathcal{S}$ for the set of all homotopy classes of non-trivial, non-peripheral simple closed curves, we can regard $\mathcal{S}$ as a subset of $\mathcal{M L}$ in the above sense. 
For $\nu \in \mathcal{M L}$ and $\alpha \in \mathcal{S}$, the intersection number $i(\nu, \alpha)$ between $\nu$ and $\alpha$ is defined to be

$$
i(\nu, \alpha)=\inf _{\alpha^{\prime}} \nu\left(\alpha^{\prime}\right)
$$

where the infimum is taken over all simple closed curves $\alpha^{\prime}$ homotopic to $\alpha$. The set $\mathcal{M L}$ is made into a topological space by the weak topology: this means that a sequence $\nu_{n}$ converges to $\nu$ in $\mathcal{M L}$ if and only if $i\left(\nu_{n}, \alpha\right)$ converges to $i(\nu, \alpha)$ for all $\alpha \in \mathcal{S}$. It is known that the intersection number function extends homogeneously and continuously on $\mathcal{M L} \times \mathcal{M L}$.

Let $\mathcal{M F}$ be the space of all measure equivalence classes of measured foliations. The set $\mathcal{S}$ can naturally be thought of as a subset of $\mathcal{M F}$. It is known that there exists a unique homeomorphism $\sigma$ from $\mathcal{M F}$ to $\mathcal{M L}$ such that the restriction to $\mathcal{S}$ is the identity. For the direct construction of $\sigma: \mathcal{M F} \rightarrow \mathcal{M L}$, we refer the reader to $\mathrm{Le}$ and also $\mathrm{Ke}$.

2.2. Teichmüller spaces. A marked Riemann surface $(Y, f)$ is a pair of a Riemann surface $Y$ and a quasiconformal mapping $f: X \rightarrow Y$. Two marked Riemann surfaces $\left(Y_{1}, f_{1}\right)$ and $\left(Y_{2}, f_{2}\right)$ are said to be Teichmüller equivalent if there exsists a conformal mapping $h: Y_{1} \rightarrow Y_{2}$ such that $h$ is homotopic to $f_{2} \circ f_{1}^{-1}$. The equivalence class of $(Y, f)$ is denoted by $[Y, f]$. The set $T(X)$ of all Teichmüller equivalence classes of marked Riemann surfaces is called the Teichmüller space of $X$. The Teichmüller distance is defined to be

$$
d_{T(X)}\left(\left[Y_{1}, f_{1}\right],\left[Y_{2}, f_{2}\right]\right):=\log \inf _{h} K(h),
$$

where the infimum is taken over all qusiconformal mappings $h: Y_{1} \rightarrow Y_{2}$ homotopic to $f_{2} \circ f_{1}^{-1}$ and the maximal dilatation of $h$ is denoted by $K(h)$. This gives $T(X)$ a complete distance function; the metric space $\left(T(X), d_{T(X)}\right)$ is homeomorphic to the open ball $\mathbb{B}^{6 g-6+2 n}$. Recall that $T(X)$ is identified with the space of all equivalence classes of hyperbolic metrics on $X$ with constant curvature -1 . Here two metrics $\rho_{1}$ and $\rho_{2}$ are said to be Teichmüller equivalent if there exists an isometry from $\left(X, \rho_{1}\right)$ to $\left(X, \rho_{2}\right)$ isotopic to the identity.

2.3. Quadratic differentials. Let $q$ be a quadratic differential on $X$ which is holomorphic except possibly at punctures. We allow the quadratic differential $q$ having a pole of order at least one at punctures. This means that the area $\|q\|=$ $\int_{X}|q(z)||d z|^{2}$ is finite, and we normalize so that the area is 1 . We refer the reader to $\mathrm{St}$ for a deeper discussion of quadratic differentials.

Each of the zeros and the poles of $q$ is called a critical point. Away from all the critical points, we define a new local coordinate, called a natural coordinate, by

$$
w=\int \sqrt{q(z)} d z
$$

for any local coordinate $z$. The transition maps are given by the form $z \mapsto \pm z+$ $c$ for $c \in \mathbb{C}$. The vertical foliation and the horizontal foliation is obtained by pulling back the vertical and the horizontal foliation, respectively, in $\mathbb{C}$ via the natural coordinates. Hence, every quadratic differential $q$ determines a pair of transverse measured foliations with singularities at the critical points of $q$; Gardiner and Masur $[\mathrm{GM}]$ showed that for any pair $(F, G)$ of transverse measured foliations with $i(F, G)=1$, there exists a unique Riemann surface $Y$ and a unique quadratic 
differential $q$ on $Y$ with unit area such that the vertical and the horizontal foliation of $q$ are measure equivalent to $F$ and $G$, respectively.

2.4. Teichmüller geodesics. Let $\nu^{+}$and $\nu^{-}$be measured laminations which fill up $X$ with $i\left(\nu^{+}, \nu^{-}\right)=1$. For each $t \in \mathbb{R}$, set $\nu_{t}^{+}=e^{t / 2} \nu^{+}$and $\nu_{t}^{-}=e^{-t / 2} \nu^{-}$. Let $\mathcal{G}_{t}$ be the Riemann surface and $q_{t}$ be the quadratic differential on $\mathcal{G}_{t}$ such that the vertical and the horizontal foliation of $q_{t}$ are measure equivalent to $\sigma^{-1}\left(\nu_{t}^{+}\right)$and $\sigma^{-1}\left(\nu_{t}^{-}\right)$, respectively. Here $\sigma$ is the homeomorphism from $\mathcal{M F}$ to $\mathcal{M L}$ as stated above. Then the path $t \mapsto \mathcal{G}_{t}$ is a geodesic on $T(X)$, and we call it the Teichmüller geodesic of $\nu^{+}$and $\nu^{-}$.

2.5. Lines of minima. It is known that for a fixed hyperbolic structure, the hyperbolic length function on $\mathcal{S}$ extends homogeneously and continuously on $\mathcal{M L}$. For any hyperbolic metric $\rho \in T(X)$, we write $\ell_{\rho}(\nu)$ for the length of a measured lamination $\nu$. Let $\nu^{+}, \nu^{-}$be as above. Kerckhoff $[\mathrm{Ke}$ showed that the length function

$$
\rho \mapsto \ell_{\rho}\left(\nu^{+}\right)+\ell_{\rho}\left(\nu^{-}\right)
$$

is minimized at a unique metric $\mathcal{L}_{0} \in T(X)$. For each $t \in \mathbb{R}$, let $\mathcal{L}_{t}$ be the unique metric which minimizes the function $\rho \mapsto e^{t / 2} \ell_{\rho}\left(\nu^{+}\right)+e^{-t / 2} \ell_{\rho}\left(\nu^{-}\right)$. Then $t \mapsto \mathcal{L}_{t}$ is a path in $T(X)$, and we call it the line of minima of $\nu^{+}$and $\nu^{-}$.

2.6. The Thurston boundary. The Thurston compactification of $T(X)$ is the closure of the Thurston embedding

$$
T(X) \ni \rho \mapsto\left[\alpha \mapsto \ell_{\rho}(\alpha)\right] \in \mathcal{P} \mathcal{R},
$$

where $\mathcal{P} \mathcal{R}=\left(\left(\mathbb{R}_{\geq 0}\right)^{\mathcal{S}}-\{0\}\right) / \mathbb{R}_{+}$. The boundary of image of $T(X)$ is called the Thurston boundary of $T(X)$. Thurston showed that the boundary coincides with $\mathcal{P} \mathcal{M}$ homeomorphic to the sphere $\mathbb{S}^{6 g-7+2 n}$; the closure of image of $T(X)$ is homeomorphic to the closed ball $\mathbb{B}^{6 g-6+2 n} \cup \mathbb{S}^{6 g-7+2 n}$. For more details we refer the reader to $\mathrm{FLP}$.

2.7. Continued fractions. We recall that every real number $\theta$ has a continuedfraction expansion of the form

$$
a_{0}+\frac{1}{a_{1}+\frac{1}{a_{2}+\frac{1}{a_{3}+\cdots}}},
$$

with $a_{0} \in \mathbb{Z}, a_{n} \in \mathbb{N}$ for $n \geq 1$ and that $\theta$ is irrational if and only if infinitely many $a_{i}$ 's are non-zero. We shall write the above expansion as $\theta=\left[a_{0} ; a_{1}, a_{2}, a_{3}, \cdots\right]$ and call $a_{n}$ an element of $\theta$. The irrational number $\theta$ is said to be of bounded type if the sequence $\left\{a_{n}\right\}_{n \in \mathbb{N}}$ is bounded and it is said to be of unbounded type if the sequence is unbounded.

For $n \in \mathbb{N}$, let $p_{n}$ and $q_{n}$ be coprime integers with

$$
\frac{p_{n}}{q_{n}}=a_{0}+\frac{1}{a_{1}+\frac{1}{a_{2}+\frac{1}{\cdots+\frac{1}{a_{n}}}}} .
$$

We denote by $\left[a_{0} ; a_{1}, a_{2}, \cdots, a_{n}\right]$ the right term in the above equation. 
The following properties [Kh] hold:

$$
\begin{gathered}
p_{n+1}=a_{n+1} p_{n}+p_{n-1} \quad \text { and } q_{n+1}=a_{n+1} q_{n}+q_{n-1}, \\
\frac{1}{q_{n}+q_{n+1}} \leq\left|p_{n}-\theta q_{n}\right| \leq \frac{1}{q_{n+1}}, \\
\frac{p_{2 n}}{q_{2 n}} \nearrow \theta \text { and } \frac{p_{2 n+1}}{q_{2 n+1}} \searrow \theta .
\end{gathered}
$$

2.8. Flat geodesics in Tori. For any $t, \theta \in \mathbb{R}$, we define the linear mapping $G_{t}^{\theta}$ by the matrix

$$
\frac{1}{\sqrt{1+\theta^{2}}}\left(\begin{array}{cc}
\theta e^{t / 2} & e^{-t / 2} \\
-e^{t / 2} & \theta e^{-t / 2}
\end{array}\right)
$$

The map $G_{t}^{\theta}$ is made of the affine mapping $\left[(x, y) \mapsto\left(e^{t / 2} x, e^{-t / 2} y\right)\right]$ followed from the rotation by the angle $\left(\pi / 2-\tan ^{-1} \theta\right)$. For any $(q, p) \in \mathbb{Z}^{2}$, we call the $(q, p)$ curve at time $t$ to be the image of $(q, p)$ under the map $G_{t}^{\theta}$, and we denote the Euclidean length of the $(q, p)$-curve at time $t$ by $l_{t}^{\theta}(q, p)$. An easy calculation shows that

$$
l_{t}^{\theta}(q, p)=\sqrt{\frac{(p-q \theta)^{2} e^{t}+(q+p \theta)^{2} e^{-t}}{1+\theta^{2}}} .
$$

Then the following lemma holds:

Lemma 2.1 (Lenzhen, $[\mathrm{L})$. Suppose that $\theta=\left[a_{0} ; a_{1}, a_{2}, a_{3}, \cdots\right]$ is an irrational number with $a_{i} \geq 3$ for $i \in \mathbb{N}$. Suppose that the $(q, p)$-curve is the shortest curve at some time $t$; that is, $l_{t}^{\theta}(q, p)=\inf l_{t}^{\theta}\left(q^{\prime}, p^{\prime}\right)$, where the infimum is taken over all $\left(q^{\prime}, p^{\prime}\right) \in \mathbb{Z}^{2}$. If $t \geq \log \left(\left(1+a_{0} \theta\right) /\left(\theta-a_{o}\right)\right)$, then $p=p_{n}$ and $q=q_{n}$ for some $n \in \mathbb{N}$. Also, at time $T_{n}=\log \left(\left(p_{n} \theta+q_{n}\right) /\left|q_{n} \theta-p_{n}\right|\right)$, the $\left(q_{n}, p_{n}\right)$-curve is the shortest. For $t \in\left[T_{n}, T_{n+1}\right]$, the shortest curve is either $\left(q_{n}, p_{n}\right)$ or $\left(q_{n+1}, p_{n+1}\right)$.

2.9. Notation. To simplify our presentation, we use the symbols $\asymp, ~ \stackrel{*}{ }, O$, and $\Theta$ defined as follows: for two functions $f, g$, the notation $f \asymp g$ and $f \stackrel{*}{\asymp} g$ mean respectively, that there are constants $c>1, C>0$ depending only on the topology of $X$ and some fixed constant $\epsilon_{0}>0$ such that

$$
\frac{1}{c} g(x)-C \leq f(x) \leq c g(x)+C \text { and } \quad \frac{1}{c} g(x) \leq f(x) \leq c g(x) .
$$

For two positive functions, $x$ and $y$, the notation $x=O(y)$ means that $x / y$ is bounded above by a constant depending only on the topology of $X$ and $\epsilon_{0}$; the notation $x=\Theta(y)$ means that $x=O(y)$ and $y=O(x)$.

\section{Minsky's PRODUCT REGION THEOREM}

In order to prove Theorem 1.1, we need to estimate the Teichmüller distance between two elements in the thin part of $T(X)$. For this purpose, Minsky's product region theorem will be useful. To state this precisely, we first introduce the notion of twists and Fenchel-Nielsen coordinates. 


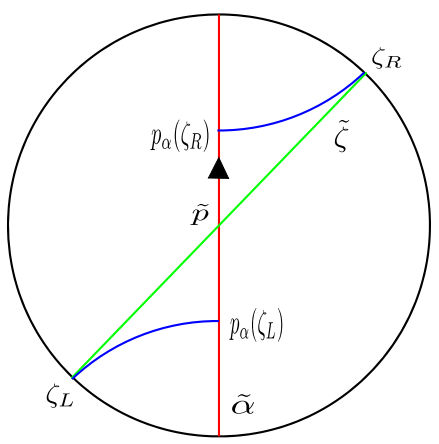

Figure 1

3.1. Twists in hyperbolic metrics. There are various ways to define twists; we shall follow Minsky Mi] and Choi, et al. CRS2]. Fix a hyperbolic metric $\rho$ on $X$. Let $\alpha$ be an oriented simple closed geodesic and let $\zeta$ be a simple geodesic that intersects $\alpha$ transversely. Let $p$ be a point of the intersection $\alpha \cap \zeta$. In the universal cover $\mathbb{D}$ of $X$, a lift $\tilde{\zeta}$ of $\zeta$ intersects a lift $\tilde{\alpha}$ of $\alpha$ at a lift $\tilde{p}$ of $p$. Let $\zeta_{R}, \zeta_{L}$ be the endpoints of $\tilde{\zeta}$ on $S^{1}$ to the right and left of $\tilde{\alpha}$, respectively. If $p_{\alpha}: \mathbb{D} \cup S_{1} \rightarrow \tilde{\alpha}$ is the orthogonal projection to $\tilde{\alpha}$, then the twist of $\zeta$ around $\alpha$ at $p$ is defined to be

$$
t w_{\rho}(\zeta, \alpha, p)= \pm \frac{d_{\mathbb{D}}\left(p_{\alpha}\left(\zeta_{R}\right), p_{\alpha}\left(\zeta_{L}\right)\right)}{\ell_{\rho}(\alpha)}
$$

where the sign is $(+)$ if the direction from $p_{\alpha}\left(\zeta_{L}\right)$ to $p_{\alpha}\left(\zeta_{R}\right)$ coincides with the orientation of $\tilde{\alpha}$ and $(-)$ if it is opposite (see Figure 1). Because different lifts of $\zeta$ are disjoint even if the simple geodesic $\zeta$ is not closed, the twist $t w_{\rho}(\zeta, \alpha, p)$ is independent of the choice of a triple $(\tilde{\zeta}, \tilde{\alpha}, \tilde{p})$ of lifts. Minsky [Mi] showed that the inequality

$$
\left|t w_{\rho}(\zeta, \alpha, q)-t w_{\rho}(\zeta, \alpha, p)\right| \leq 1
$$

holds for any point $q \in \zeta \cap \alpha$. He thus defines

$$
t w_{\rho}(\zeta, \alpha)=\min _{p \in \zeta \cap \alpha} t w_{\rho}(\zeta, \alpha, p)
$$

so that the twist is independent of the choice of a point of intersection. For a measured lamination $\nu$ that intersects $\alpha$ transversely, we can also define the twist of $\nu$ around $\alpha$ by

$$
t w_{\rho}(\nu, \alpha)=\inf _{\zeta} t w_{\rho}(\zeta, \alpha),
$$

where the infimum is taken over all leaves $\zeta$ of $\nu$. Note that the twist $t w_{\rho}(\nu, \alpha)$ depends only on the underlying geodesic lamination $|\nu|$; in other words, the transverse measure is ignored. However, we will be working with measured geodesic lamination. For convenience, we write $T w_{\rho}(\nu, \alpha)$ for $\left|t w_{\rho}(\nu, \alpha)\right|$.

3.2. Fenchel-Nielsen coordinates. Take a curve system $\left\{\alpha_{1}, \cdots, \alpha_{k}\right\}$ so that the completions of all components of $X \backslash \bigcup_{i=1}^{k} \alpha_{i}$ are pairs of pants. Recall that such a curve system is a maximal collection of disjoint, non-peripheral, non-trivial simple closed curves on $X$ and vice versa. This implies $k=3 g-3+n$. We call the system a pants curve system of $X$. Suppose, to start, that $X$ is compact (i.e., $n=0$ ). For any standard hyperbolic metric $\rho$ on $X$, we immediately obtain $k$ positive numbers 
$\left\{\ell_{\rho}\left(\alpha_{i}\right)\right\}_{i=1}^{k}$, called the length parameters. Additional $k$ real numbers, called the twist parameters, are defined as follows (see [Mi].)

A pair of pants $P$ with boundary curves $\alpha_{1}, \alpha_{2}, \alpha_{3}$ contains three unique homotopy classes of simple properly embedded arcs $\gamma_{12}, \gamma_{23}, \gamma_{13}$, called the seams, such that $\gamma_{i j}$ joins $\alpha_{i}$ to $\alpha_{j}$. Fix a set of representatives of the seams which match on opposite sides of each non-peripheral $\alpha_{i}$. This determines a curve system $\mu$ of non-trivial, non-peripheral simple closed curves.

Now for any standard hyperbolic metric $\rho$ on $X$, the seams have geodesic representatives $\gamma_{i j}^{\rho}$ which are orthogonal to $\alpha_{i}^{\rho}$ and $\alpha_{j}^{\rho}$, where $\alpha_{i}^{\rho}$ is the $\rho$-geodesic representative in the homoropy class $\alpha_{i}$. The seams cut each pair of pants into two congruent right-angled hexagons, and in particular, they bisect the boundary components. Now for each $\alpha_{j}$ and each pair of corresponding seam endpoints, there is a unique geodesic path along $\alpha_{j}$ that must be spliced between the endpoints, so that the resulting curve is homotopic to a curve in $\mu$. The length $\tau_{\rho}\left(\alpha_{j}\right)$ of this path (which can be given a sign, since $\alpha_{j}$ is oriented) is the same for both pairs of seam endpoints on $\alpha_{j}$. We define our twist parameter $s_{\rho}\left(\alpha_{j}\right)$ to be $\tau_{\rho}\left(\alpha_{j}\right) / \ell_{\rho}\left(\alpha_{j}\right)$.

This construction gives a homeomorphism $F N: T(X) \rightarrow \mathbb{R}_{+}^{k} \times \mathbb{R}^{k}$, taking $\left(\ell_{\rho}\left(\alpha_{1}\right), \ldots, \ell_{\rho}\left(\alpha_{k}\right), s_{\rho}\left(\alpha_{1}\right), \ldots, s_{\rho}\left(\alpha_{k}\right)\right)$. We note that a positive Dehn twist on $\alpha_{j}$ has the effect of incrementing $s_{\rho}\left(\alpha_{j}\right)$ by one and leaving the other coordinates invariant.

In the case with punctures, the construction is similar, except that one or two ends of a pair of pants may be a puncture, and we allow the seams to be noncompact arcs terminating in boundaries or punctures.

Minsky Mi] gives a comparison between twisting numbers and the FenchelNielsen twit parameters:

Lemma 3.1. Let $\left\{\alpha_{1}, \cdots, \alpha_{k}\right\}$ be a pants curve system of $X$ and let $\nu$ be a measured lamination on $X$ which intersects the geodesic representative of $\alpha=\alpha_{i}$ transversely for some $1 \leq i \leq k$. Then the inequality

$$
\left|\left(t w_{\rho}(\nu, \alpha)-t w_{\rho^{\prime}}(\nu, \alpha)\right)-\left(s_{\rho}(\alpha)-s_{\rho^{\prime}}(\alpha)\right)\right| \leq 4
$$

holds for any $\rho, \rho^{\prime} \in T(X)$.

3.3. Minsky's product region theorem. The Margulis lemma provides a universal constant $\epsilon_{\mathcal{M}}$ such that all components of the $\epsilon_{\mathcal{M}}$-thin part (i.e., the subset of $X$ where the injectivity radius is less than $\epsilon_{\mathcal{M}}$ ) are horocyclic neighborhoods of cusps or annular collars about short geodesics. By the $\epsilon_{\mathcal{M}}$-thick part, we mean the complement of the thin part.

Let $\mathcal{A}$ be a collection of disjoint, non-trivial, non-peripheral, simple closed curves on $X$. Adding certain curves to $\mathcal{A}$, we get a pants curve system $\mathcal{P}$ of $X$ containing $\mathcal{A}$. Let $X_{\mathcal{A}}$ be the punctured surface obtained from $X$ by removing all the curves in $\mathcal{A}$ and replacing the resulting boundary components by punctures. Note that $\mathcal{P} \backslash \mathcal{A}$ can be thought of as a pants curve system of $X_{\mathcal{A}}$.

Following [Mi], we now define a projection

$$
\Pi: T(X) \rightarrow T\left(X_{\mathcal{A}}\right) \times \mathbb{H}_{\alpha_{1}} \times \cdots \times \mathbb{H}_{\alpha_{r}},
$$

where $\mathcal{A}=\left\{\alpha_{1}, \cdots, \alpha_{r}\right\}$ and $\mathbb{H}_{\alpha_{i}}$ is the upper half-plane. The first component $\Pi_{0}$ which maps to $T\left(X_{\mathcal{A}}\right)$ is defined by forgetting the length and the twist parameters of the curves in $\mathcal{A}$ and keeping similar remaining curves in $\mathcal{P} \backslash \mathcal{A}$; the other component 
$\Pi_{\alpha_{i}}$ which maps to $\mathbb{H}_{\alpha_{i}}$ is defined by

$$
\Pi_{\alpha_{i}}(\rho)=s_{\rho}\left(\alpha_{i}\right)+i / \ell_{\rho}\left(\alpha_{i}\right) .
$$

Minsky's product region theorem states that, up to bounded additive error, the Teichmüller distance on the thin part of $T(X)$ is approximated by the sup metric on

$$
T\left(X_{\mathcal{A}}\right) \times \mathbb{H}_{\alpha_{1}} \times \cdots \times \mathbb{H}_{\alpha_{r}} .
$$

Theorem 3.2 (Minsky, [Mi]). There exists a constant $\epsilon_{0}$ smaller than $\epsilon_{\mathcal{M}}$ such that

$$
d_{T(X)}(\rho, \sigma)=\max _{\alpha \in \mathcal{A}}\left\{d_{T\left(X_{\mathcal{A}}\right)}\left(\Pi_{0}(\rho), \Pi_{0}(\sigma)\right), d_{\mathbb{H}_{\alpha}}\left(\Pi_{\alpha}(\rho), \Pi_{\alpha}(\sigma)\right)\right\} \pm O(1)
$$

holds for any $\rho, \sigma \in T_{\epsilon_{0}}(X, \mathcal{A})$. Here, by the thin part $T_{\epsilon_{0}}(X, \mathcal{A})$ of $T(X)$, we mean the subset where all the curves in $\mathcal{A}$ have hyperbolic length at most $\epsilon_{0}$.

The distance between the projections to $\mathbb{H}_{\alpha}$ can be approximated as follows:

Theorem 3.3 (Choi-Rafi-Series, CRS2). Fix a positive constant $\epsilon_{0}$ smaller than $\epsilon_{\mathcal{M}}$. Let $\alpha \in \mathcal{S}$ and let $\rho_{1}, \rho_{2} \in T_{\epsilon_{0}}(X, \alpha)$. If there exists a measured lamination $\nu$ intersecting $\alpha$ such that

$$
T w_{\rho_{i}}(\nu, \alpha) \ell_{\rho_{i}}(\alpha)=O(1)
$$

for $i=1,2$, then

$$
d_{\mathbb{H}_{\alpha}}\left(\Pi_{\alpha}\left(\rho_{1}\right), \Pi_{\alpha}\left(\rho_{2}\right)\right)=\left|\log \frac{\ell_{\rho_{1}}(\alpha)}{\ell_{\rho_{2}}(\alpha)}\right| \pm O(1)
$$

\section{Length estimates}

In this section and the next section, we introduce length estimates for short geodesics, following CRS2. All the proofs can be found in their paper, and so we omit the proofs. We see precisely in this section that the hyperbolic length of a short geodesic is comparable to the largest moduli of maximal flat annuli and maximal expanding annuli with core homotopic to the geodesic in terms of quadratic differentials (cf. Theorem 4.1); we analyze the contribution to the length of a geodesic associated to a subsurface with geodesic boundary (cf. Lemma 4.2).

4.1. Flat and expanding annuli. Let $q$ be a holomorphic quadratic differential on $X$ and let $A$ be an annulus in $(S, q)$ with piecewise smooth boundary. We say $A$ is regular if each of the boundary components $\partial_{0}, \partial_{1}$ is either non-negatively curved at every point or non-positively curved at every point and if $\partial_{0}, \partial_{1}$ are $q$-equidistant from each other. Note that a piecewise smooth curve is said to be non-negatively curved at a corner if the interior angle at the corner is at least $\pi$ and it is said to be non-negatively curved at a corner if the interior angle at the corner is at most $\pi$. Let $\kappa_{A}\left(\partial_{i}\right)$ be the integral of curvature of $\partial_{i}$ along the smooth portions, plus the sum of the interior angles at all the corners. Suppose that $A$ is a regular annulus with $\kappa_{A}\left(\partial_{0}\right) \leq 0$. We say that $A$ is an expanding annulus if $\kappa_{A}\left(\partial_{0}\right)<0$ and we call $\partial_{0}$ the inner boundary and $\partial_{1}$ the outer boundary; according to the Gauss-Bonnet theorem, we then have $\kappa_{A}\left(\partial_{1}\right)>0$. We say $A$ is a flat annulus if $\kappa_{A}\left(\partial_{0}\right)=\kappa_{A}\left(\partial_{1}\right)=0$. A regular annulus $A$ is said to be primitive with respect to $q$ if it contains no critical points of $q$ in its interior. On account of the Gauss-Bonnet theorem, every primitive annulus must either be flat or expanding. 
4.2. Modulus of annulus and the length of a short curve. Note that every simple closed curve $\alpha$ on $(S, q)$ either has a unique $q$-geodesic representative, or satisfies that all $q$-geodesic representatives foliate a flat annulus $F(\alpha)$. Note that when $\alpha$ has only one $q$-geodesic representative, $F(\alpha)$ will be thought of as a degenerate annulus which contains this geodesic alone. Let $\partial_{0}, \partial_{1}$ be the boundary components, possibly coincident, of $F(\alpha)$. Let $\hat{\partial}_{i}$ be the $q$-equidistant curve from $\partial_{i}$ outside of $F(\alpha)$ such that $\hat{\partial}_{i}$ and $\partial_{i}$ bound the largest primitive annulus $E_{i}(\alpha)$. It is possible for $\hat{\partial}_{i}$ to coincide with $\partial_{i}$. By the construction, the interior of $E_{i}(\alpha)$ is an expanding annulus with core homotopic to $\alpha$.

The following theorem holds:

Theorem 4.1. There exists a constant $\epsilon_{0}$ smaller than $\epsilon_{\mathcal{M}}$ such that

$$
\frac{1}{\ell_{\rho}(\alpha)} \asymp \max \left\{\operatorname{Mod} E_{0}(\alpha), \operatorname{Mod} F(\alpha), \operatorname{Mod} E_{1}(\alpha)\right\}
$$

holds for any $\alpha \in \mathcal{S}$ and for any $\rho \in T(X)$ with $\ell_{\rho}(\alpha)<\epsilon_{0}$.

A flat or an expanding annulus which achieves the maximum modulus in the above theorem is said to be dominant.

4.3. Length estimates on subsurfaces. Let $(X, \rho)$ be the surface $X$ with a hyperbolic metric $\rho$ and let $Q$ be a subsurface with geodesic boundary. Suppose that $\zeta$ is a simple closed geodesic in $(X, \rho)$ that intersects $\partial Q$ transversely and consider the intersection $\zeta \cap Q$ which is made up of geodesic arcs with endpoints on $\partial Q$. We can approximate $\zeta \cap Q$ by piecewise geodesic arcs homotopic to arcs in $\zeta \cap Q$, which alternately run along arcs perpendicular to $\partial Q$ and parallel to $\partial Q$. The length of the parallel portion is determined by the twist of $\zeta$ around the curves in $\partial Q$, while the portion perpendicular to $\partial Q$ is defined and estimated as explained below.

Let $\mathcal{A}$ be a collection of mutually disjoint simple closed geodesics in $(X, \rho)$. Let $Q$ be the metric completion of a connected component of $X \backslash \mathcal{A}$. Note that it is possible for two distinct boundary components of $Q$ to be identified in $S$ to a single curve in $\mathcal{A}$, so strictly speaking, $Q$ is not a subsurface. If $\eta$ is an essential geodesic arc with endpoints on $\partial Q$, let $\eta_{Q}$ be the shortest arc in $Q$ that is freely homotopic to $\eta$, relative to $\partial Q$. Hence, $\eta_{Q}$ is orthogonal to $\partial Q$.

The following lemma is a special case of Lemma 7.2 in CRS2.

Lemma 4.2. Suppose that $\ell_{\rho}\left(\alpha_{j}\right)<l$ for every component $\alpha_{j}$ of $\partial Q$ for some $l>0$. Then there exists a constant $K=K(l)$ such that for any simple closed geodesic $\zeta$ which intersects $\partial Q$ transversely,

$$
\left|\ell_{\rho}(\zeta \cap Q)-\left[\sum_{\eta} \ell_{\rho}\left(\eta_{Q}\right)+\sum_{j} \ell_{\rho}\left(\alpha_{j}\right) \frac{T w_{\rho}\left(\zeta, \alpha_{j}\right)}{2} i\left(\zeta, \alpha_{j}\right)\right]\right| \leq K i(\zeta, \partial Q),
$$

where the first sum is taken over all arcs $\eta$ in $\zeta \cap Q$ and the second sum is taken over all $j$ such that $\alpha_{j}$ intersects $\zeta$.

The Bers lemma provides a universal constant $L>0$ such that for every $\rho \in$ $T(X)$ there is a pants curve system $\mathcal{P}$ with the property that $\ell_{\rho}(\alpha)<L$ for every $\alpha \in \mathcal{P}$. We now consider the case where $Q$ is a totally geodesic pair of pants with boundary curves $\alpha_{1}, \alpha_{2}, \alpha_{3}$ of lengths $\ell\left(\alpha_{i}\right)<L$ for $i=1,2,3$. If the two endpoints of an arc $\eta$ in $\zeta \cap Q$ lie on $\alpha_{i}$ and $\alpha_{j}$, then $\eta_{Q}$ is the shortest common perpendicular 
between $\alpha_{i}$ and $\alpha_{j}$. Following Lemma 3.3 in [CRS2, we see that $\eta_{Q}$ has length $\log \left(1 / \ell\left(\alpha_{i}\right)\right)+\log \left(1 / \ell\left(\alpha_{j}\right)\right) \pm O(1)$, where the bound on the error depends only on $L$. Therefore the following estimate holds:

$$
\sum_{\eta} \ell_{\rho}\left(\eta_{Q}\right)=\sum_{j=1}^{3}\left[i\left(\zeta, \alpha_{j}\right) \log \frac{1}{\ell_{\rho}\left(\alpha_{j}\right)}\right]+O(i(\zeta, \partial Q)),
$$

where the sum is taken over all $\operatorname{arcs} \eta$ in $\zeta \cap Q$.

\section{TeichmülleR GeOdesics AND LiNES OF MiNIMA}

Fix a constant $\epsilon_{0}$ smaller than the Bers constant $L$ so that Theorems 3.2 and 4.1 are valid. For any $\rho \in T(X)$, we say a simple closed curve $\alpha$ is extremely short in $\rho$ if $\ell_{\rho}(\alpha)<\epsilon_{0}$. In this section, following CRS2, we will estimate the length and the twist parameters of extremely short curves along Teichmüller geodesics or lines of minima (cf. Theorems 5.3, 5.4, 5.5 and 5.6).

5.1. Lengths and twists along Teichmüller geodesics. For two measured laminations $\nu^{+}$and $\nu^{-}$which fill up $X$, let $\mathcal{G}=\left\{\mathcal{G}_{t}\right\}_{t \in \mathbb{R}}$ be the Teichmüller geodesic of $\nu^{+}$and $\nu^{-}$. For each $t$, let $q_{t}$ be the unique quadratic differential on $\mathcal{G}_{t}$ corresponding to the pair of $\nu_{t}^{+}$and $\nu_{t}^{-}$(cf. $\left.\S 2.3\right)$. We say $\alpha \in \mathcal{S}$ is horizontal if $i\left(\nu^{+}, \alpha\right)=0$ and it is vertical if $i\left(\nu^{-}, \alpha\right)=0$. By the balance time $t_{\alpha}$ of $\alpha$, we mean the time $t$ with the property $i\left(\nu_{t}^{+}, \alpha\right)=i\left(\nu_{t}^{-}, \alpha\right)$. We adopt the conventions that $t_{\alpha}=\infty$ if $\alpha$ is horizontal and that $t_{\alpha}=-\infty$ if $\alpha$ is vertical. Suppose that $\alpha$ is neither horizontal nor vertical, and set

$$
D_{t}(\alpha)=e^{-\left|t-t_{\alpha}\right|} d_{\alpha}\left(\nu^{+}, \nu^{-}\right),
$$

where $d_{\alpha}\left(\nu^{+}, \nu^{-}\right)$is the relative twist of $\nu^{+}$and $\nu^{-}$around $\alpha$ defined to be

$$
\inf _{\rho \in T(X)}\left(\left|t w_{\rho}\left(\nu^{+}, \alpha\right)-t w_{\rho}\left(\nu^{-}, \alpha\right)\right|\right) .
$$

Let $F_{t}(\alpha)$ be a maximal flat annulus of $q_{t}$ with core homotopic to $\alpha$. At an arbitrary time $t$, the equation

$$
\operatorname{Mod} F_{t}(\alpha)=e^{-t} \operatorname{Mod} F_{0}(\alpha)
$$

holds if $\alpha$ is vertical and the equation is the same except that the right term is replaced by $e^{t} \operatorname{Mod} F_{0}(\alpha)$ if $\alpha$ is horizontal.

The following holds for the exceptional case:

Theorem 5.1. Suppose that $\alpha \in \mathcal{S}$ is an extremely short curve in $\mathcal{G}_{t}$ which is neither vertical nor horizontal. If $F_{t}(\alpha)$ is dominant, then

$$
\operatorname{Mod} F_{t}(\alpha) \asymp D_{t}(\alpha) .
$$

On the other hand, to measure the growth of the modulus of maximal expanding annuli $E_{t}(\alpha)$, we make the following definition.

For a subsurface $Y$, there exists a unique subsurface $\hat{Y}$ with $q_{t}$-geodesic boundary in the homotopy class of $Y$ that is disjoint from the interior of $F(\alpha)$ for each boundary component $\alpha$ of $Y$. If $Y$ is not a pair of pants, define $\lambda_{Y}$ to be the length of the $q_{t}$-shortest non-peripheral simple closed curve contained in $\hat{Y}$. If $Y$ is a pair of pants, define $\lambda_{Y}$ to be $\max \left\{\ell_{q_{t}}\left(\alpha_{1}\right), \ell_{q_{t}}\left(\alpha_{2}\right), \ell_{q_{t}}\left(\alpha_{3}\right)\right\}$, where $\alpha_{i}, \alpha_{2}, \alpha_{3}$ are the three boundary curves of $\hat{Y}$ and $\ell_{q_{t}}\left(\alpha_{i}\right)$ is the length of a $q_{t}$-geodesic representative of $\alpha_{i}$ for $i=1,2,3$. 
Then the following holds:

Theorem 5.2. Suppose that $\alpha$ is an extremely short curve in $\mathcal{G}_{t}$ and $Y$ is a thick component of $\mathcal{G}_{t}$ adjacent to $\alpha$. Let $\hat{\alpha}$ be the $q_{t}$-geodesic representative of $\alpha$ on the boundary of $\hat{Y}$ and let $E_{t}(\alpha)$ be a maximal expanding annulus on the same side of $\hat{\alpha}$ as $\hat{Y}$. If $E_{t}(\alpha)$ is dominant, then

$$
\operatorname{Mod} E_{t}(\alpha) \asymp \log \frac{\lambda_{Y}}{\ell_{q_{t}}(\alpha)},
$$

where $\ell_{q_{t}}(\alpha)$ is the $q_{t}$-length of $\hat{\alpha}$.

Now we set

$$
K_{t}(\alpha)=\max \left\{\frac{\lambda_{Y_{1}}}{\ell_{q_{t}}(\alpha)}, \frac{\lambda_{Y_{2}}}{\ell_{q_{t}}(\alpha)}\right\} .
$$

As a consequence of Theorems 4.1, 5.1] and 5.2, the following theorem holds:

Theorem 5.3. Suppose that $\alpha$ is an extremely short curve in $\mathcal{G}_{t}$. If $\alpha$ is neither vertical nor horizontal, then

If $\alpha$ is vertical, then

$$
\frac{1}{\ell_{\mathcal{G}_{t}}(\alpha)} \asymp \max \left\{D_{t}(\alpha), \log K_{t}(\alpha)\right\} .
$$

$$
\frac{1}{\ell_{\mathcal{G}_{t}}(\alpha)} \asymp \max \left\{e^{-t} \operatorname{Mod} F_{0}(\alpha), \log K_{t}(\alpha)\right\} .
$$

If $\alpha$ is horizontal, the estimate is the same except that the first term is replaced by $e^{t} \operatorname{Mod} F_{0}(\alpha)$.

We need to estimate not only the lengths but also the twist parameters around short curves:

Theorem 5.4. Suppose that $\alpha$ is extremely short in $\mathcal{G}_{t}$. Then

$$
\begin{aligned}
& T w_{\mathcal{G}_{t}}\left(\nu^{+}, \alpha\right) \ell_{\mathcal{G}_{t}}(\alpha)=O(1) \text { if } t \geq t_{\alpha}, \\
& T w_{\mathcal{G}_{t}}\left(\nu^{-}, \alpha\right) \ell_{\mathcal{G}_{t}}(\alpha)=O(1) \text { if } t \leq t_{\alpha} .
\end{aligned}
$$

5.2. Lengths and twists along lines of minima. Let $\mathcal{L}=\left\{\mathcal{L}_{t}\right\}_{t \in \mathbb{R}}$ be the line of minima of $\nu^{+}$and $\nu^{-}$. Similar results for lines of minima hold:

Theorem 5.5. Suppose that $\alpha$ is an extremely short curve in $\mathcal{L}_{t}$. If $\alpha$ is neither vertical nor horizontal, then

$$
\frac{1}{\ell_{\mathcal{L}_{t}}(\alpha)} \asymp \max \left\{D_{t}(\alpha), \sqrt{K_{t}(\alpha)}\right\} .
$$

If $\alpha$ is vertical, then

$$
\frac{1}{\ell_{\mathcal{L}_{t}}(\alpha)} \asymp \max \left\{e^{-t} \operatorname{Mod} F_{0}(\alpha), \sqrt{K_{t}(\alpha)}\right\} .
$$

If $\alpha$ is horizontal, the estimate is the same except that the first term is replaced by $e^{t} \operatorname{Mod} F_{0}(\alpha)$.

Theorem 5.6. Suppose that $\alpha$ is extremely short in $\mathcal{L}_{t}$. Then

$$
\begin{aligned}
& T w_{\mathcal{L}_{t}}\left(\nu^{+}, \alpha\right) \ell_{\mathcal{L}_{t}}(\alpha)=O(1) \text { if } t \geq t_{\alpha}, \\
& T w_{\mathcal{L}_{t}}\left(\nu^{-}, \alpha\right) \ell_{\mathcal{L}_{t}}(\alpha)=O(1) \text { if } t \leq t_{\alpha} .
\end{aligned}
$$

If $\alpha$ is either vertical or horizontal, then $T w_{\mathcal{L}_{t}}\left(\nu^{+}, \alpha\right)=O(1)$ or $T w_{\mathcal{L}_{t}}\left(\nu^{-}, \alpha\right)=$ $O(1)$, respectively. 
5.3. The Teichmüller distance between $\mathcal{G}_{t}$ and $\mathcal{L}_{t}$. For $\rho \in T(X)$ and $r>0$, set

$$
\mathcal{S}_{r}(\rho)=\left\{\alpha \in \mathcal{S}: \ell_{\rho}(\alpha)<r\right\} .
$$

Then the following theorems hold:

Theorem 5.7. There exist universal constants $\epsilon<\epsilon_{0}$ such that for each $t$,

$$
\mathcal{S}_{\epsilon}\left(\mathcal{G}_{t}\right) \subset \mathcal{S}_{\epsilon_{0}}\left(\mathcal{L}_{t}\right)
$$

Theorem 5.8. Suppose that $\epsilon$ is the universal constant in the above theorem. Let $X_{0}$ be the punctured surface obtained from $X$ by removing all the curves in $\mathcal{S}_{\epsilon}\left(\mathcal{G}_{t}\right)$ and replacing the resulting boundary components by punctures. Let $\Pi_{0}$ be the map from $T(X)$ to $T\left(X_{0}\right)$ which is the projection defined by forgetting the length and the twist parameters of $\mathcal{S}_{\epsilon}\left(\mathcal{G}_{t}\right)$ and keeping the rest of the parameters (cf. §3.3). Then

$$
d_{T\left(X_{0}\right)}\left(\Pi_{0}\left(\mathcal{G}_{t}\right), \Pi_{0}\left(\mathcal{L}_{t}\right)\right)=O(1) .
$$

As a consequence of Theorems $3.2,3.3,5.4,5.6,5.7$ and 5.8 , the following formula holds:

Theorem 5.9. The Teichmüller distance between $\mathcal{G}_{t}$ and $\mathcal{L}_{t}$ is given by

$$
d_{T(X)}\left(\mathcal{G}_{t}, \mathcal{L}_{t}\right)=\max _{\alpha \in \mathcal{S}_{\epsilon}\left(\mathcal{G}_{t}\right)}\left|\log \frac{\ell_{\mathcal{G}_{t}}(\alpha)}{\ell_{\mathcal{L}_{t}}(\alpha)}\right| \pm O(1),
$$

where $\epsilon$ is the constant in Theorem 5.7. If there are no extremely short curves along $\mathcal{G}_{t}$, that is, $\mathcal{S}_{\epsilon}\left(\mathcal{G}_{t}\right)=\emptyset$ for all sufficiently large $t$, then $d_{T(X)}\left(\mathcal{G}_{t}, \mathcal{L}_{t}\right)=O(1)$.

\section{Proof of Theorem 1.1}

To begin with, following $[\mathrm{L}$, we construct an explicit quadratic differnatial. We consider the unit square in the plane $\mathbb{C}$ with the vertices $0,1,1+i$ and $i$. Take $g$ copies $U_{1}, \cdots, U_{g}$ of the square and $g$ segments $\sigma_{1}, \cdots, \sigma_{g}$, called slits, from the origin with the same length $0<s<1 / 2$ and with slopes $\theta_{1}, \cdots, \theta_{g}>0$. For each $1 \leq i \leq g$, rotating $U_{i}$ counterclockwise so that $\sigma_{i}$ is vertical, identifing every side with the opposite side in $U_{i}$ by a translation and cutting the resulting surface along $\sigma_{i}$, we get a once-holed torus $X_{i}$. The surface $X_{i}$ is naturally endowed with a flat structure. Now glue together along the slits crosswise, namely, the right side of $\sigma_{i}$ is identified with the left side of $\sigma_{i+1}$ and the left side of $\sigma_{i}$ is identified with the right side of $\sigma_{i+1}$, where $\sigma_{0}=\sigma_{g}$ and $\sigma_{g+1}=\sigma_{1}$ (see Figure 2). Let $X$ denote the resulting surface of genus $g$. Every flat surface $X_{i}$ is conformally embedded into $X$, and it will be regarded as the subsurface of $X$. Hence, the surfaces $X_{1}, \ldots, X_{g}$ give $X$ a quadratic differential with two zeros of order $(2 g-2)$. We normalize the quadratic differential so that the area is 1 , and we denote it by $q=q\left(\theta_{1}, \ldots, \theta_{g}\right)$.

From now on we make the following assumption $(*)$ :

- all of $\theta_{1}, \ldots, \theta_{g}$ are irrational numbers and

- all elements of $\theta_{i}$ are at least 3 for $1 \leq i \leq g$.

Note that Lemma 2.1 requires the above assumption.

Let $\mathcal{L}=\mathcal{L}\left(\theta_{1}, \ldots, \theta_{g}\right)$ be the lines of minima associated to $q=q\left(\theta_{1}, \ldots, \theta_{g}\right)$. We will only consider the forward direction $\mathcal{L}=\left\{\mathcal{L}_{t}\right\}_{t \geq 0}$. 


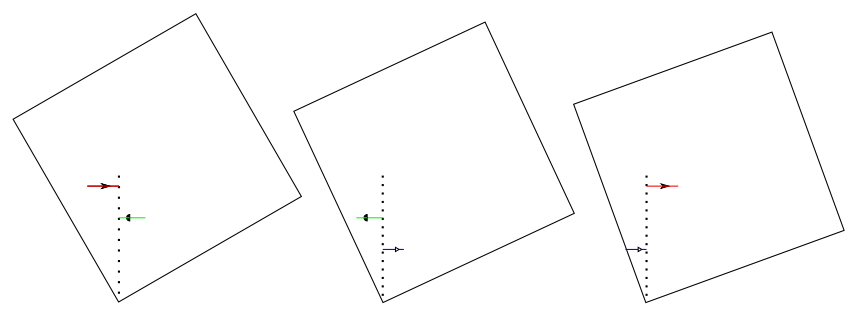

FIGURE 2. Unit squares with slits.

Theorem 1.1 is an immediate consequence of the following theorem:

Theorem 6.1. Suppose that $\theta_{1}, \ldots, \theta_{g}$ satisfy the condition $(*)$ and that $\theta_{1}$ is of bounded type and $\theta_{2}$ is of unbounded type as continued fractions. Then the line of minima $\mathcal{L}\left(\theta_{1}, \ldots, \theta_{g}\right)$ does not converge in the Thurston compactification of $T(X)$.

Throughout the proof, we will use the notation $\asymp, ~ \stackrel{*}{ }, O$ and $\Theta$ appearing in $\S 2.9$. Since we will be dealing mainly with coarse estimates, the constants in the definition of the notation need not be universal, but they must be independent of the parameter $t$.

Proof of Theorem 6.1. Suppose that the line of minima $\mathcal{L}=\left\{\mathcal{L}_{t}\right\}_{t \geq 0}$ converges to a projective measured lamination $[\nu]$ in $\mathcal{P} \mathcal{M L}$ as $t \rightarrow \infty$. By the definition of convergence in the projective space, there exists a sequence $\left\{c_{t}\right\}_{t \geq 0}$ tending to 0 such that $c_{t} \ell_{\mathcal{L}_{t}}(\gamma) \rightarrow i(\nu, \gamma)$ for any $\gamma \in \mathcal{S}$. Hence, we have

$$
\frac{\ell_{\mathcal{L}_{t}}\left(\gamma_{1}\right)}{\ell_{\mathcal{L}_{t}}\left(\gamma_{2}\right)} \longrightarrow \frac{i\left(\nu, \gamma_{1}\right)}{i\left(\nu, \gamma_{2}\right)}
$$

for any $\gamma_{1}, \gamma_{2} \in \mathcal{S}$ with $i\left(\nu, \gamma_{2}\right) \neq 0$. For the Teichmüller geodesic $\mathcal{G}=\left\{\mathcal{G}_{t}\right\}_{t \geq 0}$ of $\nu^{+}$and $\nu^{-}$, Lenzhen $\left[\mathrm{L}\right.$ showed that for any pair $\left(\theta_{i}, \theta_{j}\right)$ of a bounded type $\theta_{i}$ and an unbounded type $\theta_{j}$, there exist two sequences $t_{n}$ and $s_{n}$ tending $\infty$ and depending only on $\theta_{j}$ such that

$$
\sup _{n} \frac{\ell_{\mathcal{G}_{t_{n}}}\left(\alpha_{i}\right)}{\ell_{\mathcal{G}_{t_{n}}}\left(\alpha_{j}\right)}<\infty \text { and } \sup _{n} \frac{\ell_{\mathcal{G}_{s_{n}}}\left(\alpha_{i}\right)}{\ell_{\mathcal{G}_{s_{n}}}\left(\alpha_{j}\right)}=\infty,
$$

where $\alpha_{i}$ and $\alpha_{j}$ are the simple closed curves on $X$ corresponding to the bottom edges connecting $(0,0)$ and $(1,0)$ in $X_{i}$ and $X_{j}$, respectively. If we prove that (6) is true for $\mathcal{L}$ and that $i\left(\nu, \alpha_{i}\right) \neq 0$ for some $i$, then we get a contradiction and the proof is complete. The former assertion follows from the next lemma and the latter one follows from Theorem 7.1 appearing in the next section.

Lemma 6.2. Suppose that $\theta_{1}, \ldots, \theta_{g}$ satisfy the condition $(*)$. If $\alpha_{i}$ is the curve corresponding to the $(1,0)$-curve in $X_{i}$, then $\ell_{\mathcal{G}_{t}}\left(\alpha_{i}\right) \stackrel{*}{{ }^{*}} \ell_{\mathcal{L}_{t}}\left(\alpha_{i}\right)$.

Proof. Fix a constant $\epsilon_{0}$ smaller than the Bers constant $L$ so that Theorems 3.2 and 4.1 are valid. For simplicity of notation, we write $\theta=\theta_{1}$ and $\alpha=\alpha_{1}$ and set $\theta=\left[a_{0} ; a_{1}, a_{2}, \cdots\right]$ and $p_{n} / q_{n}=\left[a_{0} ; a_{1}, a_{2}, \cdots, a_{n}\right]$.

For each $t \in \mathbb{R}$, we consider the torus $T_{t}^{\theta}$ defined by the lattice group $\left\langle G_{t}^{\theta}(1,0), G_{t}^{\theta}(0,1)\right\rangle$, where $G_{t}^{\theta}$ is the matrix defined in $\S 2.8$. The injectivity radius $r_{i n j}\left(T_{t}^{\theta}\right)$ at a point of the torus is half of the length of the shortest $(q, p)$-curve. By 


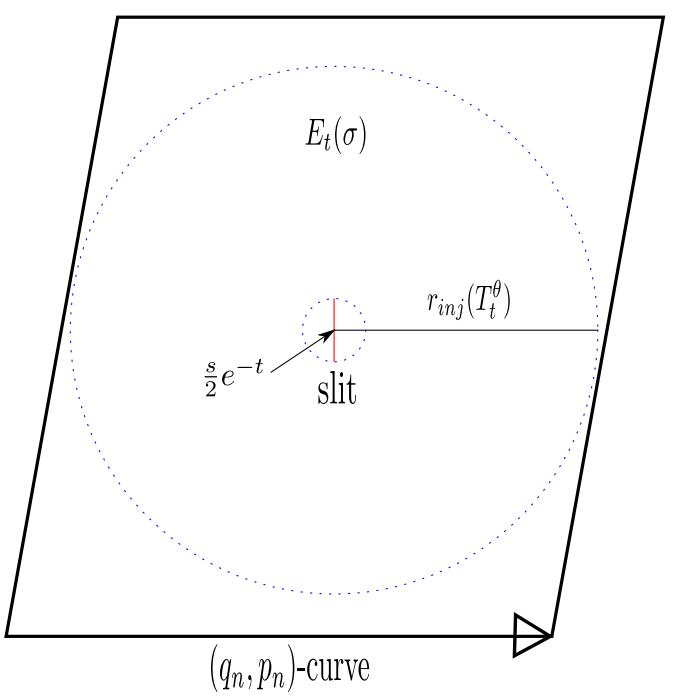

Figure 3. The expanding annulus $E_{t}(\sigma)$.

Lemma 2.1, we have $q=q_{n}, p=p_{n}$ for some $n$ and hence, $r_{i n g}\left(T_{t}^{\theta}\right)=l_{t}^{\theta}\left(q_{n}, p_{n}\right) / 2$. Let $\sigma$ be the curve on $X$ represented by the slit in $X_{1}$. Then the ring domain $E_{t}(\sigma)$ in $\mathbb{R}^{2}$ centered at the midpoint of the slit in $X_{1}$ with the inner radius $s e^{-t / 2} / 2$ and the outer radius $r_{i n g}\left(T_{t}^{\theta}\right)$ is an expanding annulus embedded into $\mathcal{G}_{t}$ with core homotopic to $\sigma$ (see Figure 3). By the geometric definition of extremal length, we get

$$
\frac{1}{\operatorname{Ext}_{\mathcal{G}_{t}}(\sigma)} \geq \operatorname{Mod} E_{t}(\sigma)=\frac{1}{2 \pi} \log \frac{l_{t}^{\theta}\left(q_{n}, p_{n}\right) / 2}{s e^{-t / 2} / 2} .
$$

From (41), we have

$$
\begin{aligned}
e^{t / 2} l_{t}^{\theta}\left(q_{n}, p_{n}\right) & =\frac{1}{\sqrt{1+\theta^{2}}} \sqrt{e^{2 t}\left(p_{n}-\theta q_{n}\right)^{2}+\left(q_{n}+\theta p_{n}\right)^{2}} \\
& \geq \frac{1}{\sqrt{1+\theta^{2}}} q_{n}\left(1+\theta \frac{p_{n}}{q_{n}}\right) .
\end{aligned}
$$

Since the right term of the above inequality goes to $\infty$ as $t \rightarrow \infty$ by the properties (11), (3), we have $\operatorname{Ext}_{\mathcal{G}_{t}}(\sigma) \rightarrow 0$ by (7). From Maskit's inequality [M], that is,

$$
\exp \left[-\ell_{\mathcal{G}_{t}}(\sigma) / 2\right] \leq \frac{\ell_{\mathcal{G}_{t}}(\sigma)}{\operatorname{Ext}_{\mathcal{G}_{t}}(\sigma)} \leq \pi
$$

we conclude that the hyperbolic length $\ell_{\mathcal{G}_{t}}(\sigma)$ of $\sigma$ also tends to zero. Therefore we deduce that the slit $\sigma$ is an extremely short curve in $\mathcal{G}_{t}$ for sufficiently large $t$.

By the Collar Lemma $[\mathrm{Bu}$, all curves with sufficiently short hyperbolic length are mutually disjoint. The homotopy class of curves $\sigma_{i}$ corresponding to the slits in $X_{i}$ are such curves and the other, if any, must be contained in $X_{i}$ for some $i$. Let $\alpha^{1}(t)$ be the unique extremely short curve at $\mathcal{G}_{t}$ contained in $X_{1}$ if any, otherwise let $\alpha^{1}(t)=\alpha$. Let $Y_{1}$ be the component of $X \backslash[\sigma]$, where $[\sigma]$ is the unique geodesic representative at $\mathcal{G}_{t}$ in the homotopy class $\sigma$, such that $Y_{1}$ is homotopy equivalent to $X_{1}$. Let $Y_{0}$ be the surface obtained from $Y_{1}$ by replacing the boundaries to punctures. Note that $Y_{0}$ is a once-punctured torus, and that every pants curve 
system is made of only one curve. Suppose that the Teichmüller space $T\left(Y_{0}\right)$ has the Fenchel-Nielsen coordinate associated to the curve $\alpha^{1}(t)$. Let the map $\Pi_{0}^{t}: T(X) \rightarrow T\left(Y_{0}\right)$ be the projection defined by keeping the length and the twist parameter of $\alpha^{1}(t)$ and forgetting the rest parameter.

For any $t$ with the condition $\alpha^{1}(t)=\alpha$, we see $d_{T\left(Y_{0}\right)}\left(\Pi_{0}^{t}\left(\mathcal{G}_{t}\right), \Pi_{0}^{t}\left(\mathcal{L}_{t}\right)\right)=O(1)$ by Theorem 5.8. From the definition of $\Pi_{0}^{t}$ and Wolpert's lemma (see Lemma 3.1 in $\mathrm{Wo}$ ), we have

$$
\frac{\ell_{\mathcal{G}_{t}}(\alpha)}{\ell_{\mathcal{L}_{t}}(\alpha)}=\frac{\ell_{\Pi_{0}^{t}\left(\mathcal{G}_{t}\right)}(\alpha)}{\ell_{\Pi_{0}^{t}\left(\mathcal{L}_{t}\right)}(\alpha)}=\Theta(1)
$$

This is the desired conclusion.

Now we assume that $\alpha^{1}(t)$ is the unique extremely short curve contained in $X_{1}$. We compute $K_{t}\left(\alpha^{1}(t)\right)$ appearing in $\S 5.1$. By the definition of $K_{t}\left(\alpha^{1}(t)\right)$, we get $K_{t}\left(\alpha^{1}(t)\right)=\max \left\{\ell_{q_{t}}(\sigma) / \ell_{q_{t}}\left(\alpha^{1}(t)\right), 1\right\}$, where $q_{t}$ is the quadratic differential corresponding to $\mathcal{G}_{t}$. For any $t \in \mathbb{R}$, there exists $n \in \mathbb{N}$ such that $\ell_{q_{t}}\left(\alpha^{1}(t)\right) \geq l_{t}^{\theta}\left(q_{n}, p_{n}\right)$ by Lemma [2.1. We conclude from the previous calculation (8) that $K_{t}\left(\alpha^{1}(t)\right)$ is bounded above. From Theorems 5.3 and 5.5, the reciprocals $1 / \ell_{\mathcal{G}_{t}}\left(\alpha^{1}(t)\right)$ and $1 / \ell_{\mathcal{L}_{t}}\left(\alpha^{1}(t)\right)$ are dominated by $D_{t}\left(\alpha^{1}(t)\right)$. Hence, we have

$$
\frac{\ell_{\Pi_{0}^{t}\left(\mathcal{G}_{t}\right)}\left(\alpha^{1}(t)\right)}{\ell_{\Pi_{0}^{t}\left(\mathcal{L}_{t}\right)}\left(\alpha^{1}(t)\right)}=\frac{\ell_{\mathcal{G}_{t}}\left(\alpha^{1}(t)\right)}{\ell_{\mathcal{L}_{t}}\left(\alpha^{1}(t)\right)}=\Theta(1) .
$$

Applying Minsky's product region theorem (Theorem 3.2$)$ to $\Pi_{0}^{t}\left(\mathcal{G}_{t}\right)$ and $\Pi_{0}^{t}\left(\mathcal{L}_{t}\right)$, we see $d_{T\left(Y_{0}\right)}\left(\Pi_{0}^{t}\left(\mathcal{G}_{t}\right), \Pi_{0}^{t}\left(\mathcal{L}_{t}\right)\right)=O(1)$ by Theorems [3.3, 5.4 and 5.6. From Wolpert's lemma, we have

$$
\frac{\ell_{\Pi_{0}^{t}\left(\mathcal{G}_{t}\right)}(\alpha)}{\ell_{\Pi_{0}^{t}\left(\mathcal{L}_{t}\right)}(\alpha)}=\Theta(1)
$$

Finally, we only need to show that

$$
\frac{\ell_{\mathcal{G}_{t}}(\alpha)}{\ell_{\Pi_{0}^{t}\left(\mathcal{G}_{t}\right)}(\alpha)}=\Theta(1) \quad \text { and } \quad \frac{\ell_{\mathcal{L}_{t}}(\alpha)}{\ell_{\Pi_{0}^{t}\left(\mathcal{L}_{t}\right)}(\alpha)}=\Theta(1)
$$

Indeed, suppose that $\rho$ is any hyperbolic metric on $X$ with the properties $\ell_{\rho}(\sigma)<\epsilon_{0}$ and $\ell_{\rho}\left(\alpha^{1}(t)\right)<\epsilon_{0}$. We will use the notation $[\gamma]$ to denote the unique $\rho$-geodesic representative homotopic to $\gamma$. Set $P=Y_{1} \backslash\left[\alpha^{1}(t)\right]$. Note that the completion of $P$ is a pair of pants. Since $[\alpha]$ is contained in $Y_{1}$, we get $\ell_{\rho}(\alpha)=\ell_{\rho}([\alpha] \cap P)$. Setting $\ell_{\rho}=\ell_{\rho}([\alpha] \cap P)$ and applying Theorem 4.2, we have

$$
\left|\ell_{\rho}-\left[\sum_{\eta} \ell_{\rho}\left(\eta_{P}\right)+\ell_{\rho}\left(\alpha^{1}(t)\right) \frac{T w_{\rho}\left(\alpha, \alpha^{1}(t)\right)}{2} i(\alpha, \partial P)\right]\right|=O(i(\alpha, \partial P)),
$$

where the sum is taken over all $\operatorname{arcs} \eta$ in $\alpha^{\rho} \cap P$. Since

$$
\sum_{\eta} \ell_{\rho}\left(\eta_{P}\right)=i(\alpha, \partial P)\left(\log \frac{1}{\ell_{\rho}\left(\alpha^{1}(t)\right)}+O(1)\right)
$$

by (5), we get

$$
\left|\frac{\ell_{\rho}}{i(\alpha, \partial P)}-\left[\log \frac{1}{\ell_{\rho}\left(\alpha^{1}(t)\right)}+\ell_{\rho}\left(\alpha^{1}(t)\right) \frac{T w_{\rho}\left(\alpha, \alpha^{1}(t)\right)}{2}\right]\right|=O(1) .
$$


For $\delta>0$, let $\Pi_{\delta}^{t}(\rho)$ be the element in $T(X)$ such that the length parameter of $\sigma$ is equal to $\delta$ and the rest of the parameters coincide with those of $\rho$. Because of $\ell_{\mathcal{G}_{t}}\left(\alpha^{1}(t)\right)=\ell_{\Pi_{\delta}^{t}\left(\mathcal{G}_{t}\right)}\left(\alpha^{1}(t)\right)$ by the definition of $\Pi_{\delta}^{t}$, we see

$$
\log \frac{1}{\ell_{\mathcal{G}_{t}}\left(\alpha^{1}(t)\right)}=\log \frac{1}{\ell_{\Pi_{\delta}^{t}\left(\mathcal{G}_{t}\right)}\left(\alpha^{1}(t)\right)} .
$$

Moreover, because of $s_{\mathcal{G}_{t}}\left(\alpha^{1}(t)\right)=s_{\Pi_{\delta}^{t}\left(\mathcal{G}_{t}\right)}\left(\alpha^{1}(t)\right)$, we also see

$$
\left|\left(t w_{\mathcal{G}_{t}}\left(\alpha, \alpha^{1}(t)\right)-t w_{\mathcal{G}_{0}}\left(\alpha, \alpha^{1}(t)\right)\right)-\left(s_{\mathcal{G}_{t}}\left(\alpha^{1}(t)\right)-s_{\mathcal{G}_{0}}\left(\alpha^{1}(t)\right)\right)\right| \leq 4
$$

and

$$
\left|\left(t w_{\Pi_{\delta}^{t}\left(\mathcal{G}_{t}\right)}\left(\alpha, \alpha^{1}(t)\right)-t w_{\mathcal{G}_{0}}\left(\alpha, \alpha^{1}(t)\right)\right)-\left(s_{\mathcal{G}_{t}}\left(\alpha^{1}(t)\right)-s_{\mathcal{G}_{0}}\left(\alpha^{1}(t)\right)\right)\right| \leq 4
$$

from Lemma 3.1. and hence,

$$
\left|T w_{\mathcal{G}_{t}}\left(\alpha, \alpha^{1}(t)\right)-T w_{\Pi_{\delta}^{t}\left(\mathcal{G}_{t}\right)}\left(\alpha, \alpha^{1}(t)\right)\right| \leq 8 .
$$

Combining (9), (10) and (11), we get

$$
\frac{\ell_{\mathcal{G}_{t}}(\alpha)}{\ell_{\Pi_{\delta}^{t}\left(\mathcal{G}_{t}\right)}(\alpha)}=\Theta(1)
$$

Letting $\delta \rightarrow 0$, we conclude that

$$
\frac{\ell_{\mathcal{G}_{t}}(\alpha)}{\ell_{\Pi_{0}^{t}\left(\mathcal{G}_{t}\right)}(\alpha)}=\Theta(1)
$$

Applying the same argument to $\mathcal{L}_{t}$, we can get the same conclusion for $\mathcal{L}_{t}$. It follows that $\ell_{\mathcal{G}_{t}}\left(\alpha_{1}\right) \stackrel{*}{=} \ell_{\mathcal{L}_{t}}\left(\alpha_{1}\right)$ as required.

\section{The Limit SET OF $\mathcal{L}$}

Let $\nu^{+}$and $\nu^{-}$be the measured laminations corresponding to the vertical foliation $F$ of $q$ and the horizontal foliation $G$ of $q$, respectively. Since $F$ has the closed leaf $\sigma_{i}$ which is the closed curve on $X$ corresponding to the slit of the slope $\theta_{i}$, the underlying geodesic lamination of $\nu^{+}$also has a closed leaf $\left[\sigma_{i}\right]$ homotopic to $\sigma_{i}$ (see [Le]), whereas the transverse measure of $\nu^{+}$is not supported on $\left[\sigma_{i}\right]$. Let $Y_{1}, \ldots, Y_{g}, Q$ be components of $X \backslash \bigcup_{i=1}^{g}\left[\sigma_{i}\right]$, where $Y_{i}$ is the component homotopy equivalent to $X_{i}$ (see Figure 4). Note that $Q$ is the complement of the completion of $Y_{1} \cup \cdots \cup Y_{g}$ and that $Q=\emptyset$ when $g=2$. Then $\nu^{+}$can be divided into sublaminations $\nu_{1}^{+}, \ldots, \nu_{g}^{+}$supported on $Y_{1}, \ldots, Y_{g}$, respectively. We write $\nu^{+}=\nu_{1}^{+}+\cdots+\nu_{g}^{+}$.

Let $\mathcal{L}$ be the line of minima in the previous section and let $A(\mathcal{L})$ be the set of all accumulation points of $\mathcal{L}$ in the Thurston compactification. We call the set $A(\mathcal{L}) \backslash \mathcal{L}$ the limit set of $\mathcal{L}$ and we denote it by $L(\mathcal{L})$. Since $\mathcal{L}$ is proper, $L(\mathcal{L})$ must be a subset of $\mathcal{P} \mathcal{M L}$.

The following theorem and corollary hold:

Theorem 7.1. Suppose that $\theta_{1}, \ldots, \theta_{g}$ satisfy the condition $(*)$ and that $\theta_{1}$ is of bounded type as a continued fraction. Then $L(\mathcal{L})$ is a closed connected subset of the $(g-1)$-dimensional simplex

$$
\left\{\left[a_{1} \nu_{1}^{+}+\cdots+a_{g} \nu_{g}^{+}\right]: a_{i} \geq 0 \text { for } 1 \leq i \leq g, \sum_{i=1}^{g} a_{i}=1\right\} .
$$




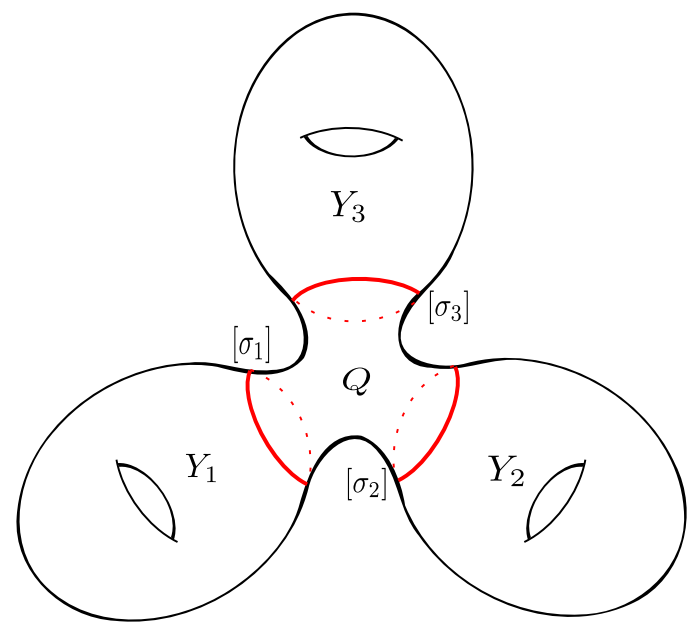

FIGURE 4

Corollary 7.2. Under the same condition of Theorem 6.1, if $\theta_{2}=\cdots=\theta_{g}$, then $\left[\nu_{1}^{+}\right] \in L(\mathcal{L})$. Therefore if $g=2$, the limit set $L(\mathcal{L})$ is a segment, one of whose endpoints is $\left[\nu_{1}^{+}\right]$.

Similar results of the above theorem and corollary are true for the Teichmüller geodesic associated to Lenzhen's quadratic differential (see $[\mathrm{L}]$ ).

Proof of Theorem 7.1. Suppose that $[\nu]$ is an accumulation point of $\mathcal{L}=\left\{\mathcal{L}_{t}\right\}_{t \geq 0}$ in $\mathcal{P} \mathcal{M L}$. There exist sequences $\left\{t_{n}\right\}_{n \in \mathbb{N}}$ tending to $\infty$ and $\left\{c_{n}\right\}_{n \in \mathbb{N}}$ tending to zero such that

$$
c_{n} \ell_{\mathcal{L}_{t_{n}}}(\xi) \rightarrow i(\nu, \xi)
$$

for any $\xi \in \mathcal{M L}$.

Since

$$
\ell_{\mathcal{L}_{t}}\left(\nu^{+}\right) \leq \ell_{\mathcal{L}_{t}}\left(\nu^{+}\right)+e^{-t} \ell_{\mathcal{L}_{t}}\left(\nu^{-}\right) \leq \ell_{\rho}\left(\nu^{+}\right)+e^{-t} \ell_{\rho}\left(\nu^{-}\right)
$$

for all $\rho \in T(X)$ by the definition of $\mathcal{L}_{t}$, we have $\ell_{\mathcal{L}_{t}}\left(\nu^{+}\right)=O(1)$. We get $i\left(\nu, \nu^{+}\right)=$ 0 from (12) and hence, $i\left(\nu, \nu_{i}^{+}\right)=0$ for all $1 \leq i \leq g$. Let $\sigma_{i}$ be the closed curve corresponding to the slit in $X_{i}$. Since $\ell_{\mathcal{L}_{t}}\left(\sigma_{i}\right) \rightarrow 0$ as $t \rightarrow \infty$, we have $i\left(\nu, \sigma_{i}\right)=0$ from (12). This implies that the geodesic representative $\left[\sigma_{i}\right]$ of $\sigma_{i}$ and the underlying geodesic lamination $|\nu|$ of $\nu$ do not intersect transversely. Hence,

$$
\nu=\sum_{i=1}^{g}\left(a_{i} \nu_{i}^{+}+b_{i} \sigma_{i}\right)+\mu,
$$

where $a_{1}, \ldots, a_{g}$ and $b_{1}, \ldots, b_{g}$ are non-negative numbers and $\mu$ is the maximal sublamination supported on $Q$.

It suffices to show that $b_{1}=\cdots=b_{g}=0$ and $|\mu|=\emptyset$. To prove this, we need to estimate the hyperbolic length of a curve $\gamma_{i}$ across both of $\sigma_{1}$ and $\sigma_{i}$ for each $2 \leq i \leq g$. For instance, take $\gamma_{i}$ as the curve made of $\alpha_{1}$ followed by $\alpha_{i}$ (see Figure 51). We will use the notation $\left[\gamma_{i}\right]$ to denote the unique geodesic in the homotopy class of $\gamma_{i}$. 


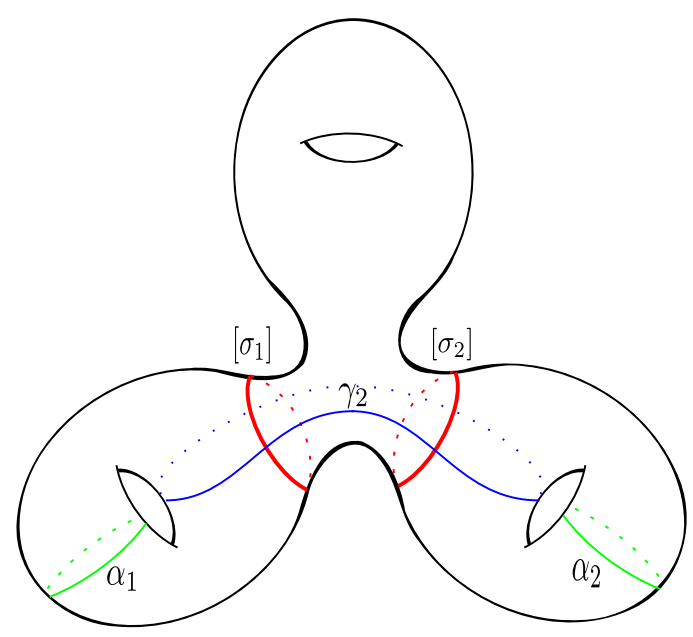

Figure 5

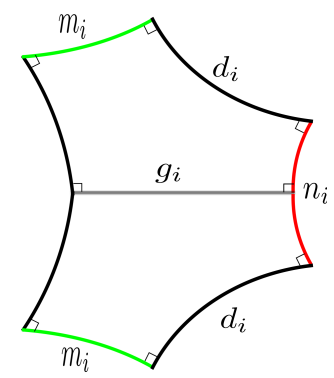

Figure 6 . Half of the pair of pants $P_{i}$.

For simplicity of notation, we write $\ell_{\mathcal{L}_{t}}(\cdot)$ for $\ell(\cdot)$. Set $m_{i}=\ell\left(\alpha_{i}\right) / 2$ and $n_{i}=$ $\ell\left(\sigma_{i}\right) / 2$. Set $P_{i}=Y_{i} \backslash\left[\alpha_{i}\right]$. Applying the cosine formula for right-angled hexagons Bu] to $P_{i}$, we have,

$$
\cosh d_{i}=\frac{\cosh m_{i}\left(1+\cosh n_{i}\right)}{\sinh m_{i} \sinh n_{i}}
$$

where $d_{i}$ is the length of a common perpendicular between $\left[\sigma_{i}\right]$ and $\left[\alpha_{i}\right]$ contained in $P_{i}$ (see Figure 6). Since $m_{i} \rightarrow \infty$ and $n_{i} \rightarrow 0$ as $t \rightarrow \infty$, we have

$$
\frac{\cosh m_{i}}{\sinh m_{i}} \rightarrow 1, \quad \frac{\sinh n_{i}}{n_{i}} \rightarrow 1 \quad \text { and } \quad \cosh n_{i} \rightarrow 1
$$

Thus, we get

$$
\cosh d_{i}=2 \varepsilon_{1} / n_{i},
$$

where $\varepsilon_{1} \rightarrow 1$. Applying the cosine formula for right-angled pentagons $\left[\mathrm{Bu}\right.$ ] to $P_{i}$, we have

$$
\cosh g_{i}=\sinh m_{i} \sinh d_{i},
$$


where $g_{i}$ is half of the length of a perpendicular $\delta_{i}$ between $\left[\sigma_{i}\right]$ and itself contained in $P_{i}$. We then have

$$
g_{i}=\log \left(\cosh g_{i}+\sinh g_{i}\right)=m_{i}+\log \left(1 / n_{i}\right)+\varepsilon_{2},
$$

where $\varepsilon_{2} \rightarrow 0$. Let $s\left(\sigma_{i}\right)$ be the twist parameter of $\sigma_{i}$ for $\mathcal{L}_{t}$. It follows that

$$
2 g_{i} \leq \ell\left([\gamma] \cap P_{i}\right) \leq 2 g_{i}+2 \ell\left(\sigma_{i}\right)\left|s\left(\sigma_{i}\right)\right| .
$$

We conclude from Lemma 3.1 and Theorem 5.6 that $\ell\left(\left[\gamma_{i}\right] \cap P_{i}\right)=2 g_{i}+O(1)$, and hence, from (13) that

$$
\ell\left(\left[\gamma_{i}\right] \cap P_{i}\right)=\ell\left(\alpha_{i}\right)+2 \log \left(1 / \ell\left(\sigma_{i}\right)\right)+O(1) .
$$

On the other hand, let $\eta_{i}$ be the shortest $\operatorname{arc}$ in $Q$ which connects $\left[\sigma_{1}\right]$ and $\left[\sigma_{i}\right]$. We denote the hyperbolic length of $\eta_{i}$ by $h_{i}$. Since the $q$-geodesic representative $\hat{Q}$ of $Q$ is the union of all slits, we have $K_{t}(\beta) \leq 1$ for all non-peripheral curves $\beta$ contained in $Q$. Since maximal flat annuli of $\beta$ are degenerate, we have $\operatorname{Mod} F_{0}(\beta)=0$. Therefore we conclude that there is no extremely short curve contained in $Q$ by Theorem 5.5. Note that a thick component of $X$ has a bounded geometry; that is, the diameter of the thick component is bounded for any hyperbolic metric on $X$. This gives

$$
h_{i}=\log \frac{1}{\ell\left(\sigma_{1}\right)}+\log \frac{1}{\ell\left(\sigma_{i}\right)}+O(1)
$$

It follows that

$$
2 h_{i} \leq \ell\left(\left[\gamma_{i}\right] \cap Q\right) \leq 2 h_{i}+2 \ell\left(\sigma_{1}\right)\left|s\left(\sigma_{1}\right)\right|+2 \ell\left(\sigma_{i}\right)\left|s\left(\sigma_{i}\right)\right| .
$$

We conclude from Lemma 3.1 and Theorem 5.6 that $\ell\left(\left[\gamma_{i}\right] \cap Q\right)=2 h_{i}+O(1)$; hence, from (15),

$$
\ell\left(\left[\gamma_{i}\right] \cap Q\right)=2 \log \frac{1}{\ell\left(\sigma_{1}\right)}+2 \log \frac{1}{\ell\left(\sigma_{i}\right)}+O(1) .
$$

Since $\ell\left(\gamma_{i}\right)=\ell\left(\left[\gamma_{i}\right] \cap P_{1}\right)+\ell\left(\left[\gamma_{i}\right] \cap Q\right)+\ell\left([\gamma] \cap P_{i}\right)$, combining (14) and (16), we deduce that

$$
\ell_{\mathcal{L}_{t}}\left(\gamma_{i}\right)=\ell_{\mathcal{L}_{t}}\left(\alpha_{1}\right)+\ell_{\mathcal{L}_{t}}\left(\alpha_{i}\right)+4\left(\log \frac{1}{\ell_{\mathcal{L}_{t}}\left(\sigma_{1}\right)}+\log \frac{1}{\ell_{\mathcal{L}_{t}}\left(\sigma_{i}\right)}\right)+O(1) .
$$

Now we claim that

$$
\frac{\ell_{\mathcal{L}_{t}}\left(\gamma_{i}\right)}{\ell_{\mathcal{L}_{t}}\left(\alpha_{1}\right)+\ell_{\mathcal{L}_{t}}\left(\alpha_{i}\right)} \rightarrow 1
$$

Indeed, since there is no flat annulus with core homotopic to $\sigma_{i}$ with respect to the quadratic differential $q_{t}$ corresponding to $\mathcal{G}_{t}$, the reciprocals of $\ell_{\mathcal{G}_{t}}\left(\sigma_{i}\right)$ and $\ell_{\mathcal{L}_{t}}\left(\sigma_{i}\right)$ are dominated by the terms of $K_{t}\left(\sigma_{i}\right)$ in Theorems 5.3 and 5.5 . Hence, we have

$$
\log \frac{1}{\ell_{\mathcal{L}_{t}}\left(\sigma_{i}\right)} \stackrel{*}{`} \frac{1}{\ell_{\mathcal{G}_{t}}\left(\sigma_{i}\right)} .
$$

It follows from Lemma 6.2 that

$$
\frac{\log \left(1 / \ell_{\mathcal{L}_{t}}\left(\sigma_{i}\right)\right)}{\ell_{\mathcal{L}_{t}}\left(\alpha_{1}\right)+\ell_{\mathcal{L}_{t}}\left(\alpha_{i}\right)} \stackrel{*}{\longleftarrow} \frac{1 / \ell_{\mathcal{G}_{t}}\left(\sigma_{i}\right)}{\ell_{\mathcal{G}_{t}}\left(\alpha_{1}\right)+\ell_{\mathcal{G}_{t}}\left(\alpha_{i}\right)} .
$$

Since $\hat{Q}$ is degenerate and $\lambda_{X_{i}}=O(1)$, by Lemma 2.1 we see that $K_{t}\left(\sigma_{i}\right)=O\left(e^{t / 2}\right)$, and hence, that $1 / \ell_{\mathcal{G}_{t}}\left(\sigma_{i}\right)=O(t)$. Let $\beta_{1}(t)$ be the Euclidean shortest $(q, p)$-curve 
in $X_{1}$ at time $t$. By Lemma 2.1, we have $p=p_{n}$ and $q=q_{n}$ for some $n$, where $\theta_{1}=\left[a_{0} ; a_{1}, a_{2}, \ldots\right]$ and $p_{k} / q_{k}=\left[a_{0} ; a_{1}, \cdots, a_{k}\right]$. Again by Lemma 2.1 we see

$$
t \leq \log \frac{p_{n+1} \theta_{1}+q_{n+1}}{\left|q_{n+1} \theta_{1}-p_{n+1}\right|} .
$$

An easy computation by using the properties (11) and (2) shows that

$$
\frac{p_{n+1} \theta_{1}+q_{n+1}}{\left|q_{n+1} \theta_{1}-p_{n+1}\right|} \leq\left(1+\theta_{1} \frac{p_{n+1}}{q_{n+1}}\right)\left(a_{n+1}+2\right) q_{n+1}^{2} .
$$

Because of the boundedness of $\left\{a_{n}\right\}_{n \in \mathbb{N}}$, we must have $t=O\left(\log q_{n}\right)$. On the other hand, since the collar around $\beta_{1}(t)$ is bounded, we have $\ell_{\mathcal{G}_{t}}\left(\alpha_{1}\right) \geq \Theta\left(i\left(\alpha_{1}, \beta_{1}(t)\right)\right)=$ $\Theta\left(p_{n}\right)=\Theta\left(q_{n}\right)$. Therefore we deduce that

$$
\frac{1 / \ell_{\mathcal{G}_{t}}\left(\sigma_{i}\right)}{\ell_{\mathcal{G}_{t}}\left(\alpha_{1}\right)+\ell_{\mathcal{G}_{t}}\left(\alpha_{i}\right)} \leq \frac{1 / \ell_{\mathcal{G}_{t}}\left(\sigma_{i}\right)}{\ell_{\mathcal{G}_{t}}\left(\alpha_{1}\right)} \leq \frac{O\left(\log q_{n}\right)}{\Theta\left(q_{n}\right)} \longrightarrow 0 .
$$

From (17), (20) and (21), it might be concluded that (18) is valid. It follows that $b_{1}=b_{i}=0$ and $i\left(\mu, \gamma_{i}\right)=0$ for each $2 \leq i \leq g$. Since a component of $Q \backslash \bigcup_{i=2}^{g}\left(\left[\gamma_{i}\right] \cap Q\right)$ is a geodesic polygon, it is simply connected and finally we deduce that $\mu=\emptyset$.

Because of the continuity of the function $\mathbb{R}_{\geq 0} \ni t \longmapsto \mathcal{L}_{t} \in T(X)$, the limit set $L(\mathcal{L})$ must be closed and connected.

Proof of Corollary 17.2. It follows from Lemma 6.2 that (6) holds for $\mathcal{L}$. There exists a sequence $\left\{s_{n}\right\}_{n \in \mathbb{N}}$ tending to $\infty$ and depending only on $\theta_{2}=\cdots=\theta_{g}$ such that

$$
\frac{\ell_{\mathcal{L}_{s_{n}}}\left(\alpha_{1}\right)}{\ell_{\mathcal{L}_{s_{n}}}\left(\alpha_{i}\right)} \rightarrow \infty
$$

for any $2 \leq i \leq g$. Let $[\nu]$ be an accumulation point of $\left\{\mathcal{L}_{s_{n}}\right\}_{n \in \mathbb{N}}$. By Theorem 7.1, we see that $\nu=\sum_{i=1}^{g} a_{i} \nu_{i}^{+}$for some $a_{1}, \ldots, a_{g} \geq 0$ with $\sum_{i=1}^{g} a_{i}=1$. Hence, (22) shows that $a_{i}=0$ for $2 \leq i \leq g$. Therefore $[\nu]=\left[\nu_{1}^{+}\right]$.

Remark. Suppose that $\theta_{1}, \ldots, \theta_{g}$ satisfy the condition $(*)$. It follows from (19) and Wolpert's lemma that $d_{T(X)}\left(\mathcal{G}_{t}, \mathcal{L}_{t}\right)$ goes to $\infty$. However, the proof of Lemma 6.2 shows that $d_{T\left(X_{0}\right)}\left(\Pi_{0}\left(\mathcal{G}_{t}\right), \Pi_{0}\left(\mathcal{L}_{t}\right)\right)$ is bounded above. Here $X_{0}$ is the surface obtained from $X$ by removing all curves corresponding to the slits and replacing the boundaries to punctures and $\Pi_{0}$ is the forgotten map from $T(X)$ to $T\left(X_{0}\right)$. In general, we are interested in finding a condition when $d_{T(X)}\left(\mathcal{G}_{t}, \mathcal{L}_{t}\right)$ is bounded, where $\mathcal{G}_{t}$ and $\mathcal{L}_{t}$ are induced by an arbitrary quadratic differential (cf. Theorem [5.9).

\section{ACKNOWLEDGMENT}

The author would like to thank Professor Hiroshige Shiga for several helpful comments.

\section{REFERENCES}

[Bu] P. Buser, Geometry and spectra of compact Riemann surfaces. Progress in Mathematics 106, Birkhäuser, Boston (1992). MR1183224 (93g:58149)

[CRS1] Y. Choi, K. Rafi and C. Series, Lines of minima are uniformly quasigeodesics, Pacific J. Math. 237:1 (2008), 21-44. MR2415205 (2009b:32017) 
[CRS2] _ _ Lines of minima and Teichmüller geodesics. Geom. Funct. Anal. 18 (2008), 698754. MR2438996 (2010k:30053)

[DS] R. Diaz and C. Series, Limit points of lines of minima in Thurston's boundary of Teichmüller space. Alg. Geom. Top. 3 (2003), 207-234. MR1997320 (2004f:32014)

[FLP] A. Fathi, F. Laudenbach, and V. Poénaru, Travaux de Thurston sur les surfaces. Astérisque, Vol. 66-67, Soc. Math. de France (1979). MR568308 (82m:57003)

[GM] F. Gardiner and H. Masur, Extremal length geometry of Teichmüller space. Complex Variables Theory Appl. 16 (1991), no. 2-3, 209-237. MR.1099913 (92f:32034)

[Ke] S. Kerckhoff, Lines of minima in Teichmüller space. Duke Math. J. 65 (1992), no. 2, 187-213. MR 1150583 (93b:32027)

[Kh] A. Khinchin, Continued fractions. Russian edition, Dover Publications, Mineola, NY (1997). MR:1451873 (98c:11008)

[L] A. Lenzhen, Teichmüller geodesics that do not have a limit in $\mathcal{P} \mathcal{M F}$. Geom. and Top. 12 (2008), 177-197. MR2377248(2008k:30056)

[Le] G. Levitt, Foliations and laminations on hyperbolic surfaces. Topology 22 (1983), 119-135. MR683752 (84h:57015)

[M] B. Maskit, Comparison of extremal and hyperbolic lengths. Ann. Acad. Sci. Fenn. 10 (1985), 381-386. MR802500 (87c:30062)

[Ma] H. Masur, Two boundaries of Teichmüller space. Duke Math. J. 49 (1982), 183-190. MR650376 (83k:32035)

[Mi] Y. Minsky, Extremal length estimates and product regions in Teichmüller space. Duke Math. J. 83 (1996), 249-286. MR 1390649 (97b:32019)

[St] K. Strebel, Quadratic Differentials, Springer Verlag, Berlin and New York (1984). MR743423 (86a:30072)

[Wo] S. Wolpert, The length spectra as moduli for compact Riemann surfaces. Ann. Math. 109 (1979), 323-351. MR528966 (80j:58067)

Department of Mathematics, Tokyo Institute of Technology, Oh-okayama, MeguroKU, TOKYO 152-8551, JAPAN

E-mail address: iguchi.y.ac@m.titech.ac.jp 